OPEN ACCESS

UWS Academic Portal

\title{
A multi-site survey of forensic nursing assessment
}

Walker, Helen; Tulloch, Lindsay; Boa, Karen; Ritchie, Gordon ; Thomson, John

Published in:

Journal of Forensic Practice

DOI:

10.1108/JFP-11-2018-0045

Published: 13/05/2019

Document Version

Peer reviewed version

Link to publication on the UWS Academic Portal

Citation for published version (APA):

Walker, H., Tulloch, L., Boa, K., Ritchie, G., \& Thomson, J. (2019). A multi-site survey of forensic nursing assessment. Journal of Forensic Practice, 21(2), 124-138. https://doi.org/10.1108/JFP-11-2018-0045

\section{General rights}

Copyright and moral rights for the publications made accessible in the UWS Academic Portal are retained by the authors and/or other copyright owners and it is a condition of accessing publications that users recognise and abide by the legal requirements associated with these rights. 
OPEN ACCESS

\section{UWS Academic Portal}

\section{A multi-site survey of forensic nursing assessment}

Walker, Helen; Tulloch, Lindsay; Boa, Karen; Ritchie, Gordon ; Thomson, John

Published in:

Journal of Forensic Practice

Accepted/In press: 28/02/2019

Document Version

Peer reviewed version

Link to publication on the UWS Academic Portal

Citation for published version (APA):

Walker, H., Tulloch, L., Boa, K., Ritchie, G., \& Thomson, J. (Accepted/In press). A multi-site survey of forensic nursing assessment. Journal of Forensic Practice.

\section{General rights}

Copyright and moral rights for the publications made accessible in the UWS Academic Portal are retained by the authors and/or other copyright owners and it is a condition of accessing publications that users recognise and abide by the legal requirements associated with these rights.

This is an Open Access item distributed under the terms of the Creative Commons Attribution-NonCommercial-NoDerivatives License (http://creativecommons.org/licenses/by-nc-nd/4.0/), which permits non-commercial re-use, distribution, and reproduction in any medium, provided the original work is properly cited, and is not altered, transformed, or built upon in any way.

Take down policy

If you believe that this document breaches copyright please contact us providing details, and we will remove access to the work immediately and investigate your claim. 


\section{A multi-site survey of forensic nursing assessment}

\begin{tabular}{|r|l|}
\hline Journal: & Journal of Forensic Practice \\
\hline Manuscript ID & JFP-11-2018-0045.R2 \\
\hline Manuscript Type: & Research Paper \\
\hline Keywords: & $\begin{array}{l}\text { Risk assessment, Behavioural Status Index, Forensic, Care Planning, } \\
\text { Secure hospital, Nursing assessment }\end{array}$ \\
\hline \multicolumn{2}{|l}{} \\
\hline
\end{tabular}

\section{SCHOLARONE \\ Manuscripts}


Title: A multi-site survey of forensic nursing assessment - (5,561 words) new changes marked in red

\section{Introduction}

In clinical practice the imbalance of safety and security versus therapeutic engagement and recovery creates tension and often leads to risk-aversion. Nowhere is the tension more keenly felt than in forensic services, where the patients are by definition more prone to engage in high risk behaviours that pose severe challenges to maintaining the safety of all concerned (Drennan and Alred, 2012). The consequences of managing serious violent or sexual risks may reduce the possibility for collaboration and empowerment and the possibility of patients remaining in hospital for long periods increases the likelihood of institutionalisation (Corlett and Miles, 2010). For the reasons highlighted above it is important to find an assessment process that is suitable and acceptable for both patients and staff alike, supported by decision making methods that relate to risk assessment and management (Doyle and Dolan, 2002).

Assessment of behaviour is an ongoing process that nurses undertake on a daily basis, often in order to aid the development of individual care plans (Olsson and Schon, 2016). It is recognised that behaviour change is an indicator of the likelihood and severity of risk behaviours occurring, and observing clinicians tend to use this as a measure to gauge the patients' ability to function socially. In former years it has been suggested that improvement in social functioning was the key to recovery in psychosis (Turkington and Bryant, 2007); this is especially important in forensic services where over 70\% suffer from psychotic illnesses (Darjee et al., 2017; Vojt et al., 2011; Thomson et al., 1997). A major difficulty in the past was a shortage of appropriate instruments with which nurses could carry out valid and reliable therapeutic assessments, were behaviourally based and therefore appropriate for use in a variety of contexts (Woods, Reed and Robinson, 1999). Risk assessment scales now exist in abundance, for example, the Historical, Clinical and Risk Management -20 ([HCR-20] Webster et al, 1997, Douglas et al., 2014) and the Short-Term Assessment of Risk and Treatability ([START] Webster et al., 2009). Whist nurses do provide information for these instruments and they are certainly useful 
for measuring social risk for violence, the information provided on therapeutic outcomes and core areas of therapeutic activity over defined periods of time is more limited (Ross et al., 2012). There are other scales that concentrate on violent behaviour, such as the Staff Observation Aggression Scale Revised ([SOAS-R] Nijman et al., 1999) or the antecedents of violent behaviour, for example, the Broset violence checklist ([BVC] Woods and Almvik, 2002) and the Dynamic Assessment of Situational Aggression ([DASA] Ogloff and Daffern, 2006), but they do not focus on daily living skills.

Over a decade ago a need for change in the way patients are assessed was identified through a review of mental health nursing in Scotland (Scottish Executive Health Department, 2006). In keeping with the national drive to support recovery-oriented care, in 2007 a programme of work began to introduce a new and comprehensive system for nursing assessment, care planning and report writing within The State Hospital (TSH) a high secure facility in Scotland. This same system was subsequently adopted by National Forensic Mental Health Services (NFMHS) Ireland, a high and medium secure service, and Murray Royal Hospital (MRH), a medium and low secure service in Scotland. As part of this development an assessment tool was introduced across the three sites, Behavioural Status Index [BEST-Index] (Reed and Woods, 2000).

The BEST-Index (Reed and Woods, 2000) was designed for people with psychosis in forensic services which gives it a distinct advantage over other measures. It incorporates several sub-scales and in its current form assesses six key areas of behaviour, see Box 1, building on the original work of Mahgoub (1989). The aim is to identify, describe and categorise behaviours associated with or potentially predictive of risk (Woods, Reed and Collins, 2001b).

\section{Insert Box 1 here}

BEST-Index intends to help clinicians establish systematic baselines for important life skills performance in patients; provide individual patient data for use in treatment planning; allow precise treatment planning goals to be set, and facilitate systematic documentation of re-measures for 
purposes of monitoring health outcomes (Woods, Reed and Collins, 2003). It was also designed to aid assessment in a variety of psychiatric contexts and provide data to inform therapeutic interventions (Woods, Reed and Collins, 2001a). In Scottish services patients tend to move from one level of security to another (for example high to medium or medium to low secure care) as they progress towards reintegration in the community. The versatility of a tool was deemed essential as was the necessity to generate robust data that could be used to plan care with individual patients. The wider multidisciplinary assessment tool routinely used is the Historical Clinical Risk assessment (HCR-20 V3; Douglas et al., 2014) which uses current and historical behaviour to examine the patient against a range of pathological indexes (Chakhassi et al., 2010); earlier research identified a close correlation between BEST-Index and the dynamic factors of the HCR-20 (Ross et al., 2008). The HCR-20 V3 (Douglas et al., 2014) is currently in use across all Scottish forensic services.

BEST-Index has been subject to psychometric testing to assess its validity and reliability over the past few decades (Woods, Reed and Collins, 2003; Ross et al., 2008; Chakhssi, de Ruiter and Bernstein, 2010; Ross et al., 2012). Despite use in the research context, there is limited evidence of BEST-Index being actively applied and tested in routine clinical practice, and few reports of patients or staff views of the tool other than Sukkoo et al. (2007). The inclusion of patient perspective was considered important because their contributions can add a new dimension to risk assessment. Arguably if risk assessment and management are to be truly effective they should be undertaken in the spirit of collaboration with the goal of helping patients become better assessors and managers of their own risk (Cree and Horstead, 2013).

\section{Aims}

This project aimed to ascertain the utility of the BEST-Index with patients in high, medium and low forensic services through: 
- exploring if there is any changing pattern in patients' social skills as they pass through the forensic network services as measured by the clinical assessment tool BEST-Index;

- establishing patients impression of the assessment tool, and

- exploring the utility of the instrument as part of routine clinical practice - through discussion with multi-disciplinary staff.

\section{Methods}

A repeated measures cross-sectional survey was carried out over an eighteen month period. A mixed method approach using complementary quantitative and qualitative data was used to gather data. It is thought that mixed method studies capitalize on the strengths of each quantitative and qualitative method, thus researchers can produce stronger and more credible studies that can yield both complementary and corroborating evidence about the research problem of interest (Plano Clark and Ivancova, 2016). The assessment tool BEST-Index generated quantitative data. One to one interviews were used to gather patient opinion due to the lower refusal rate (Parahoo, 2014), potential to gather rich data (Polit and Hungler, 1993) and ability to provide a platform to promote an understanding of the complex social, behavioural and educational issues of the patients' life experiences (Press, 2004). However careful consideration had to be afforded to the time constraints of the project, costs for researcher's time and the potentially high number of respondents. For these reasons questionnaires were also used to gather qualitative data from clinicians.

A triangulated approach was taken to gain a meaningful impression of the utility in clinical practice, incorporating three strands of work. The first strand focussed on tracking change, the second on patient impression and third on staff opinion. These three elements were explored across all three research sites. A brief summary of qualitative findings are reported as part of this article; the focus is predominantly on quantitative results. 


\section{Ethical consideration}

The study was approved by TSH Research Committee and complied with required ethical and legal standards, relating to participant confidentiality and anonymity. No patient was contacted until consent was issued from the relevant Consultant Psychiatrist. Patients and staff were given written information about the project and given seven days to consider their participation, they were assured that they could withdraw at any time. Patients were advised that their care and treatment would not be affected in any way should they decline the invitation to take part. Written informed consent was obtained from all participants. All data generated from the research was kept in a locked cabinet in a secure unit within TSH. Data transported from other sites had unique identifiers attached to forms rather than names and was transferred in a locked briefcase with security code only recognised by the Chief (HW) and Principal Investigators (LT, GR, JT). All notes and data from interviews with patients and staff were anonymised, removing all identifiable identification prior to analysis and sharing with the research team.

\section{Element 1. Tracking change}

The following hypothesis was tested:

- There will be a difference in the degree of social competence and social risk that patients present with as they pass through the forensic network services; as measured by BEST-Index. The assessment was employed at baseline and at an 18 month follow up interval to measure potential change in patient behavior. The sample included patients across all three sites who had been assessed using BEST-Index within three months of the baseline date; all were recruited by the respective Research Assistants at each site, following consent from Consultant Psychiatrists. 


\section{Assessment scale}

\section{Behavioural Status Index (BEST-Index) (Reed and Woods, 2000)}

BEST-Index is arguably one of the most appropriate and acceptable behavioural assessment tools for nurses in forensic psychiatry (Woods, Reed and Collins, 2003). The BEST-Index relies on information gathered from several sources - through observation, discussion with patients, colleagues and review of case notes. It was first introduced to TSH in 2002 as part of an international research project (Ross et al., 2008).

All items are rated using the Likert scale, on a scale of 1-5, with higher scores indicating a higher level of functioning thus a high score on the risk subscale is suggestive of a lower level of risk behaviours. A nurse (or other practitioner) familiar with the patient - alongside the patient themselves - rates the items (Woods, Reed and Collins, 2001b). Three sub-scales have 30 items to be rated (empathy, communication and social skills, self and family care) and three have 20 items (risk, insight, work and recreation). The range of a 20 item sub-scale is $20-100$ and a 30 item sub-scale is $30-150$. Each item is criterion referenced, which adds to the objectivity of the scale. The range of the total score is minimum 150 and maximum is 750 .

\section{Data analysis}

Quantitative data generated from the scores from BEST-Index was entered into SPSS version 19 (SPSS Inc, 2012) analysed and compared across sites. Each subscale was pro-rated to account for missing data; scales with more than $20 \%$ of the data missing were excluded from each analysis using the formula: $S_{P}=\left(S_{t} \times N\right) /(N-n) S_{P}\left(S_{P}=\right.$ prorated score; $S_{t}=$ total score for subscale; $N=$ total number of items in scale; $n=$ number of missing items) (Chu et al., 2013). Data analysis of demographic characteristics included measures of central tendency (mean, mode and median) and dispersion (range, standard deviation). Tests of normality of distribution (Kolmogorov-Smirnof, $p=.20$ and Shapiro-Wilk, $p=.23$ ) and tests for equality of variance (Levene's, $p=.28$ ) were employed at baseline 
on total scores, all were non significant. Parametric tests (paired sample $t$ tests) were then used to measure change tracked over two time points. Individual patient scores were compared at time one (T1) baseline and time two (T2) eighteen month follow up. Clinical change is deemed to occur when there is movement of one point for $20 \%$ of any one sub-scale e.g. improvement of 4 points on a 20 item sub- scale (see Fig 1 for an example of Risk sub-scale). This was agreed through collaboration with one of the original authors Professor Val Reed.

\section{Insert Fig 1 here}

\section{Element 2. Patient impression}

Semi-structured interviews were carried out with a 1:4 purposive sample across all sites at baseline. Purposive sampling has been used extensively in clinical research and is deemed acceptable methodologically (Parahoo, 2014). It allows for the selection of participants with relevant knowledge and expertise, enabling researchers to be better informed on the topic under review. An advert was sent out to all Key Workers (Registered Nurses) asking if they could recommend any of their patients for participation in the study. Responses were sent to the Research Assistants at the respective sites, thereafter consent to approach the patient was sought from the Consultant Psychiatrist.

\section{Sample}

Patients were included if they were aged between $18-65$ and had a diagnosis of mental disorder or learning disability. They were excluded if they were too unwell to consent, as agreed by their Consultant Psychiatrist.

On agreed dates a sample of patients were selected for interview from TSH ( $n=30)$, NFMHS $(n=30)$ and from MRH $(n=16)$. All participants were selected on the basis of their ability to answer questions and availability on the agreed dates. The interviews were undertaken by a Research Assistant from each of the three sites - all of whom were trained in the same interview technique by HW (Chief 
Investigator) and GR (Principal Investigator) in advance of the interviews. Interviews across all sites were co-ordinated by HW. Total number of interviews ( $n=76)$.

\section{Research questions}

- Do patients value the opportunity to take an active role in the assessment and care planning process?

- What do patients think about the assessment tool BEST-Index selected for use across forensic services?

- Do the individual care plans written by key workers reflect the strengths and needs of the patient?

The interview required participants to answer a small number of fixed response questions in addition to open-ended questions. Some questions posed within the interview were presented to both staff and patients.

\section{Element 3. Staff opinion}

Two similar staff questionnaires were developed by the research team, predominantly using fixed responses on a five point Likert scale, but with optional sections for additional comment. Staff were asked to complete the questionnaires only once at baseline. Questionnaire (a) was issued to all Medical, Occupational Therapy, Psychology, Team Leaders/Charge Nurses, Senior Charge Nurses and Social Work staff working at each site. Questionnaire (b) was issued to Key Workers (KW).

\section{Research questions}

- Are members of the multi-disciplinary team (MDT) familiar with BEST-Index assessment tool?

- Do the members of the multi-disciplinary team value BEST-Index 


\section{Data analysis}

Qualitative data generated from the patient interviews and staff questionnaires (elements two and three) were analysed using thematic analysis. This is a method for identifying, and interpreting central themes emerging from the data (Taylor et al., 2010). The advantages of this type of analysis are that it is flexible and can provide a rich and detailed account of the data (Braun \& Clarke 2006). The main aim of the analysis was to uncover key themes reflecting participants' opinions. The qualitative analysis was initially conducted by two researchers (HW and LT). Responses to each question from the interview transcripts were studied separately in turn, with responses reread numerous times to identify key features. Initial categories containing similar responses were then gathered together and sub divided into emerging themes. Themes were then studied and interpreted. In keeping with this methodology, themes were based on the prevalence of particular patterns within the data, but also in terms of relevance and importance of these patterns to the research aims (Braun and Clarke 2006). Finally, once the themes were generated the validity in relation to the data set was considered. This ensured that the themes reflected an accurate representation of the data which was transparent to the reader. The two researchers discussed the results until a list of themes was mutually agreed upon; very few discrepancies occurred. Rigour was achieved from the engagement with participants and the breadth and depth of data collection. Results emerging from these elements of the project will not be reported in full, only a summary will be offered.

\section{Results}

\section{Survey results}

The total sample included participants from three sites, TSH $(n=134), \operatorname{NFMHS~}(n=50)$ and MRH $(n=61)$, a total sample size of $(n=245)$. Descriptive statistics were only collected for the sample of patients who were interviewed $(n=76)$, not the entire sample $(n=245)$, due to time and resource constraints. The 
participants were predominantly male $(n=75,98 \%)$, with a mean age of 40 years (standard deviation $(S D)=10.67$, range, 20-65). The majority were single $(n=57,75 \%)$, others were either married/cohabiting $(n=3,4 \%)$, divorced / separated $(n=11,15 \%)$, or widowed $(n=5,6 \%)$. The majority of participants were caucasian and had been unemployed prior to admission ( $n=55,72 \%)$, a few worked in professional field ( $n=6,7 \%)$, the remainder in a variety of manual trades, shop sales roles, students or were retired $(n=16,21 \%)$. A common range of diagnoses was evident including schizophrenia, schizoaffective, paranoid schizophrenia, bipolar disorder and depressive disorder.

\section{Tracking change}

\section{Changing patterns of behaviour}

The hypothesis that BEST-Index can detect clinical improvement or deterioration in social competence and social risk can be accepted because there were statistically significant differences in scores over the two time periods in three of the BEST-Index subscales (risk, insight, work and recreation), all of which indicated improvement, see Table 1. Effect sizes, calculated using Cohen's $d$ are considered 'small' when $d=0.2$ (Brace, 2008), meaning the difference between the two groups was minimal despite reaching statistical significance (see Table 1). A further two subscales (Communication and Self-care) also improved although did not reach statistical significance. Empathy differed from the others and reduced over the time period, although not to a significant degree.

\section{Table 1 insert here}

There is also a statistically significant improvement in total Scottish BEST-Index scores when moving through the different levels of security - from TSH (high secure) at baseline ( $n=118, m=490$, $s d=106$ ) and 18 month follow up $(n=71, m=520, s d=110)$ to $M R H$ (medium secure) service at baseline $(n=50$, $m=570, s d=119)$ and 18 month follow up $(n=31, m=571, s d=109)$ - see Table 2. Effect size was calculated and a medium effect (0.7) was noted when comparing high and medium secure total scores at baseline. These findings from the two Scottish units provide new baseline information for future 
comparison. Clinical change (using the marker of $20 \%$ improvement in scores) was evident in ( $n=58$, $36 \%)$ of the total sample in Social Risk; $(n=60,39 \%)$ in Communication; $(n=75,50 \%)$ in Self Care; $(n=77$, $48 \%$ ) in Insight; ( $n=73,48 \%$ ) in Work and Recreation and ( $n=69,46 \%$ ) in Empathy. Clinical deterioration was also evident, but in a smaller proportion of patients: ( $n=38,24 \%)$ Social Risk; ( $n=38$, $25 \%)$ in Communication; $(n=35,23 \%)$ in Self Care; $(n=29,18 \%)$ in Insight; $(n=35,25 \%)$ in Work and Recreation and ( $n=42,28 \%)$ in Empathy. Others patients remained fairly static.

Tests of reliability

Inter - rater reliability was tested on the first $(n=100)$ results, a reasonable degree of reliability was found between all sub scales. The average measure Intraclass Correlation (ICC) was .62, with a $95 \%$ confidence interval from .38 to $.76(F(99,495)=4.66, p<.001$. Tests for internal consistency on the full sample found Cronbach alpha coefficients for the scales ranging from .90 - .97 (.90 risk, .96 insight, .94 communication, .94 work and recreation, .94 self care and .97 empathy) demonstrating excellent internal consistency, showing that the items within the tool are closely related. This is a measure of scale reliability. This is on a par with earlier research by Woods, Reed and Collins (2003), which found coefficients between .90 and .96. This result should be viewed with caution because the tool is a 150 item schedule and the number of test items can inflate the Cronbach's alpha if there is redundancy or duplication.

\section{Table 2 insert here}

Interview results

The views of $(n=76)$ participants are presented on a topic that seemed to generate varied levels of interest. Patient impression centred around one overarching theme of 'Acceptance of the process'; sub themes emerged on the 'level of awareness' , 'how involved we felt in the process' and the 'importance assessment and care planning holds for us'. Sub themes generated from the interviews are highlighted below in italics. 


\section{Level of awareness}

$\mathrm{N}=7,9 \%$ had absolutely no idea what nursing assessment was about. One participant (Participant 10) simply thought it indicated how he was 'coping with day to day life', another (Participant 12) said he had 'no idea' and supposed it was just about his Key Worker (KW) taking notes about his presentation 'and whether I am assaultive or not'. Most of those asked ( $n=54,71 \%)$ had a fair grasp of the concept of nursing assessment, understanding it was based on nursing observation of their behaviour and mental state and that the information would be used to help plan their care. A further $(n=15,20 \%)$, believed it to be an assessment the KW makes which forms the basis for a report that goes to the clinical team, the report can be used for intermediate and annual reviews and may include both positive and negative information.

How involved we felt in the process

In relation to the level of involvement the patients have, the majority ( $n=55,72 \%)$ were actively involved and had regular (commonly weekly) discussions with their KW and received their annual reports. A few (Participant 3 and 18) commented on the 'advantage of having as associate worker during periods when my KW is on night duty or on holiday'. The Scottish KW system includes the use of an associate worker as part of the team for each patient, the associate worker assumes the KW role in their absence. $\mathrm{N}=20,26 \%$ thought the involvement was 'not bad', although one (Participant 16) felt he was 'not well enough involved to comment properly' another (Participant 9) stated that he 'actually was not really that interested' and another (Participant 4) said he would often just 'listen to what the KW says'. Only one (Participant 12) did not feel at all involved in the process.

Importance assessment and care planning holds for us

Level of importance afforded to the assessment and subsequent care planning process again provoked a positive response. $\mathrm{N}=37,49 \%$ noted it was very important to patients for a variety of reasons, they: want to know where they need to improve; want to know what is happening; want to be able to 
express their opinion and feel involved and want to know what you have to do to move on. Others ( $n=38,50 \%)$ thought it was quite important.

'because you can make judgements on when you think you are likely to get things, such as grounds access' (Participant 14).

It seemed less of an issue for patients who were likely to be in hospital for a lengthy period as a consequence of their tariff.

'there is no urgency to find out where I'm going' (Participant 7).

'I don't care, they just write what they want regardless of my view' (Participant 12).

\section{Staff opinion}

\section{Demographic details: Lead Nurses, Senior Charge Nurses and Nursing Team Leaders}

There were $(n=9,64 \%)$ females and $(n=5,36 \%)$ males, the majority $(n=13,93 \%)$ aged between $30-54$ years and had been working in the services for many years.

\section{Staff Nurses / Key Workers}

Feedback was received from a total of $(n=188)$ participants, with an even split between female $(n=92$, $49 \%)$ and male ( $n=96,51 \%)$. This indicates approximately $40 \%$ return rate from all units. Responses were received from a group of staff with considerable experience in the forensic service, see Figure 2.

\section{Insert Fig $\mathbf{2}$ here}

\section{Multi-disciplinary Team Members (MDT)}

A total of $(n=70)$ responses were received across three sites, male $(n=28,40 \%)$, female $(n=42,60 \%)$, including a variety of disciplines. This represents a reasonable proportion, approximately $55 \%$ of the MDT population. Age of participants and length of service did not vary significantly across the three sites, see Figure 3. 


\section{Insert Fig 3 here}

There was a variable length of service, notably longer service in NFMHS with considerable length of experience in forensic services, see Figure 4.

\section{Insert Fig 4 here}

The one overarching theme emerging from the MDT and Nursing staff questionnaires was

'Production and delivery of information'. This was broken down into three sub themes including, 'comprehension', 'confidence to apply the tool in practice' and 'contribution'.

\section{Comprehension}

Some members of the MDT were unfamiliar with the BEST- Index tool $(n=11,16 \%)$, and during team meetings did not know what the scores meant.

'I was left confused because I didn't know how to interpret the scores, I was new and it wasn't explained' (Participant 10).

It was noted more than once that nurses do not always explain the tools when presenting their reports at MDT meetings and that it would be helpful to the wider group if they did. The vast majority of staff nurses were comfortable with the level of understanding they had on the tool and felt able to transfer the information onto care plans. A small group still thought they are not familiar enough with the BEST-Index tool to use it effectively and would like refresher training.

'I did receive training in the tool but I am only key worker for one patient, so don't use it that often....I just maybe need some reassurance that I am completing it properly and maybe a quick update would help' (Participant 3). 
Confidence to apply the tool in practice

Although many nurses commented they were happy as long as they had time to prepare, some feel less comfortable and confident delivering their report to the MDT, due to the infrequence of undertaking this exercise.

‘I sometimes feel a bit uncomfortable.... especially when we have to do our report last..... it feels like everything has already been said, so I just read out what I've written down' (Participant 14).

The practical application of translating the output from the assessment into a care plan enabled staff to focus more readily on evidence based practice.

'I feel more confident writing care plans and am comfortable with a strengths base approach' (Participant 8).

Contribution

The senior nursing staff definitely valued the nursing contribution and could see the benefit of using such an assessment tool.

'I like it because it is broad ranging and robust and can identify the need for therapeutic interventions which nurses can subsequently participate in' (Participant 4).

Unfortunately some of the MDT members were unable to see how the BEST-Index nursing assessment linked to the wider multi-disciplinary assessment; others were very clear about its place in the process.

'the BEST- Index is useful because of the correlation between it and many of the HCR-20 items' (Participant 3).

The majority of nurses were content.

'I feel as though we have something useful to report at long last' (Participant 22). 
Yet a few felt their contribution was not valued.

'half the time it seems that nobody even listens to our feedback at the MDT' (Participant 1).

Perhaps this was directly attributable to the lack of understanding about the tool and level of communication offered to enhance understanding of the application to the wider multi-disciplinary agenda.

\section{Discussion}

The utility of BEST-Index was examined across three forensic services. The hypothesis indicated there would be a difference in the degree of social competence and social risk patients present with as they pass through the forensic network services. BEST-Index has formerly demonstrated clinical utility and can be administered repeatedly during treatment to assess changes in multiple dimensions of forensic risk (Chakhssi, de Ruiter and Bernstein, 2010). This study further supports the sensitivity of the tool, demonstrating statistically significant improvement in socially acceptable behaviour between patients passing from high to medium secure services. Results do have to be viewed with caution due to the small effect sizes. One of the six sub scales (empathy) highlighted deterioration rather than improvement, in keeping with findings from the original research study (Ross et al., 2008). One possible explanation for this may be a result of patients having to spend prolonged periods of time in the company of others (not through choice) who present with challenging behaviours periodically. Ross et al. $(2012$, p386) maintain that 'profound knowledge of patient behaviour is a precondition for both risk assessment and treatment planning '. Indeed, the ability to assess, assimilate the information gathered, and subsequently manage risky behaviours is fundamental to forensic nurses. In order to do this with a degree of consistency it could be argued that the use of a structured assessment tool is not only desirable but essential. It is accepted that the use of standardised assessment tools enables clinicians to gauge the likelihood of future violence in an individual, in that, they can identify the problems and needs a person may have (National 
Institute for Clinical Excellence [NICE], 2007) and subsequently manage future risk of harm (Vojt et al., 2011).

Patient impression and staff opinion of the BEST-Index assessment tool and subsequent care planning processes were also explored, revealing both positive and negative issues. It would seem that awareness of the tool is fairly good across patients and staff alike, but could be improved by further training and better communication and collaboration with members of the wider MDT.

A more collaborative approach to assessment and care planning with patients is likely to foster positive therapeutic relationships and develop trust (Bee et al, 2015), it can also potentially enhance relational security. It is important to consider the barriers and enablers to individual involvement in care if recovery is to be successful. Where there is poor information exchange, this can create a major barrier to involvement in patient's care planning (Bee et al., 2015). Working in partnership with patients to undertake assessment and aid planning of care may always pose a challenge in forensic services regardless of the method or tools utilised for this purpose, because attitudes towards such tasks are not always positive (Drennan and Alred, 2012). The impression from the majority of patients was that they valued the process of assessment and subsequent planning of their care - to a point - but it was not something that held great interest for them. Making small adjustments to practice to address the issues highlighted earlier can lead to a more confident nursing workforce, which may subsequently improve team cohesion and ultimately patient care. The impression from the majority is that the assessment tool has good clinical utility.

\section{Limitations}

The methodology was selected for pragmatic reasons. There are known limitations with crosssectional surveys, namely that they are restricted by gathering data at given time points and may miss relevant information. The selection process used to identify participants was purposive rather than random sampling, which does reduce the degree of objectivity and has the potential for a biased subject group. Although semi structured interview was undertaken by experienced 
researchers, patients seemed somewhat disinterested in this subject area, thus did not always actively engage. One plausible reason for the disinterest could be the considerable number of projects participants engage in over the years. The consequence of this limited report by patients is the absence of potentially pertinent issues. The number of responses in the final sample was affected by large volumes of missing data, radically reducing the sample size. This impacts on the generalisability of the findings. Finally, only half of the subscales demonstrated statistically significant improvement; this coupled with the small effect size perhaps indicates limited sensitivity to detect change.

\section{Conclusion}

In conclusion, this study has served to highlight some of the advantages and disadvantages of the current nursing assessment tool used across forensic services and its application in practice. Feedback from patients has highlighted both good and poor practice across all three sites. It would seem that improved collaboration with patients at the assessment stage can ease the process of care planning. The views of nurses including more senior staff was generally quite positive, but some multidisciplinary members clearly had issues with the assessment and reporting processes. It would seem there was still a considerable amount of work to be done to raise awareness and subsequently improve impact. The tool itself proved to be fairly robust and have a degree of clinical utility. We now have data to suggest that clinical improvement was evident in a proportion of patients (ranging from $36 \%-50 \%$ across the various sub-scales) over an eighteen month period in this forensic population. There was also a statistically significant improvement in total Scottish BEST-Index scores when moving through the different levels of security, demonstrating a degree of sensitivity of the tool. These results serve to give a baseline for future clinical practice. 
Implications for practice:

- results gave confidence in the utility of the tool, providing evidence to support its ability to capture behavior change through different levels of security, continued use is therefore recommended;

- due to concerns linked to limited awareness of the tool (from a small group), a strategy to improve information sharing for patients and staff was established;

- the importance of good communication across disciplines needs to be more effective, especially if nursing assessment (BEST-Index) continues to be used to inform the wider MDT risk assessment (HCR-20 V3);

- feedback from patients regarding their assessment and care planning suggests the majority want to be involved and a more collaborative approach could perhaps improve future therapeutic engagement;

- in MRH there was a distinct drop in empathy at 18 month follow up and baseline scores seemed artificially high, there was therefore a need to revisit scoring of empathy scale;

- in NFMHS communication and empathy reduced, reasons for this led to local exploration.

\section{References}

Addington, J., Addington, D. (2008) Outcome after discharge from an early psychosis program. Schizophrenia Research, 106 (2-3) 363-366.

Bee, P., Brooks, H., Fraser, C. and Lovell, K. (2015) Professional perspectives on service user and carer involvement in mental health care planning: A qualitative study. International Journal of Nursing Studies, 52 (12) 1834-1845.

Bowling, A. (2014) Research methods in health; Investigation health and health services. Berkshire: Open University Press.

Brace, N., Kemp, R. and Snelgar, R. (2009) SPSS for Psychologists, $4^{\text {th }}$ edition. Hampshire: Palgrave MacMillan.

Braun, V. and Clark, V. (2006) Using thematic analysis in psychology. Qualitative research in psychology, 3 (2) 77-101. 
Brace, N., Kemp, R. and Snelgar, R. (2009) SPSS for Psychologists 4h Edition. London: Palgrave MacMillan.

Chakhssi, F, de Ruiter, C, Bernstein, D. (2010) Reliability and Validity of the Dutch version of the behavioural status index: a nurse rater forensic assessment tool. Assessment, 17(1) 58-69.

Chu, C, Thomas, D, Ogloff, J, Daffern, M. (2013) The Short to Medium Term Predictive Accuracy of Static and Dynamic Risk Assessment Measures in a Secure Forensic Hospital. Assessment, 20 (230).

Clark, M. (2015) Co-production in mental health care. Mental Health Review Journal, 20 (4). ISSN 1361-9322.

Corlett, H. and Miles, H. (2010) An evaluation of the implementation of the recovery philosophy in a secure forensic service. British Journal of Forensic Practice, 12 (4) 14-25.

Cree, A. and Horstead, A., (2013) Achieving transparency in forensic risk assessment: a multimodal approach. Advances in Psychiatric Treatment, 19 (5) 351-357.

Darjee, R., Ofstegaard, M., Thomson, L.D.T. (2017) Schizophrenia in a high-security hospital: longterm forensic, clinical, administrative \& social outcomes. Journal of Forensic Psychiatry and Psychology, 28 (4) 525-547. doi.org/10.1080/14789949.2017.1308537

Douglas, K.S., Hart, S.D., Webster, C.D., Belfrage, H., Guy, L,S., \& Wilson, C.M. (2014) Historical-Clinical-Risk Management-20, Version 3 (HCR-20V3): Development and Overview International Journal Forensic Mental Health, 13 (2) 93-108. doi.10.1080/14999013.2014.906519

Doyle, M. and Dolan, M. (2002) Violence risk assessment: combining actuarial and clinical information to structure clinical judgements for the formulation and management of risk. Journal of Psychiatric and Mental Health Nursing, 9, 649-657 doi: 10. 1046/j.1365-2850.2002.00535.x

Drennan, G. and Alred, D. (Eds) (2012) Secure Recovery; Approaches to recovery in forensic mental health settings. London, Willey and Sons.

Gale, J. and Marshall-Lucette, S. (2012) Community mental health nurses' perspectives of recoveryoriented practice. Journal of Psychiatric and Mental Health Nursing, 19, 348-353.

Mahgoub, N.R. (1989) Bridging therapy in hospital and community based psychiatric nursing care: a comprehensive study (two volumes). Unpublished PhD Thesis, (sponsoring establishment Sheffield City Polytechnic), Council for National Academic Awards.

NICE (2007) Best Practice in Managing Risk: Principles and Evidence for Best Practice in the Assessment and Management of Risk to Self and Others in Mental Health. London: NICE.

Nijman, H.L.I., Muris, P., Merckelbach, H.L.G.J., Palmstierna, T., Wistedt, B., Vos, A.M., et al. (1999) The staff observation aggression scale-revised (SOAS-R) Aggressive Behaviour, (25) 197-209. doi: 10.1002/(SICI)1098-2337(1999)25:3<197::AID-AB4>3.0.CO;2-C.

Ogloff, J. and Daffern, M. (2006) The dynamic appraisal of situational aggression: an instrument to assess risk for imminent aggression in psychiatric patients. Behavioural Science and Law, 24 (6) 799813. 
Olsson, H. and Schon, U-K. (2016) Reducing violence in forensic care - how does it resemble the domains of a recovery-oriented care? Journal of Mental Health, 25 (6) 506-511. Doi:10.3109/09638237.2016.1139075

Parahoo, K. (2014) Nursing Research; Principles, Process and Issues (3 ${ }^{\text {rd }}$ Edition) London: Palgrave MacMillan.

Polit, D.E. and Hungler, B.P. (1993) Essentials of Nursing Research: Method, Appraisal an Utilisation $3^{\text {rd }}$

Edition Philadelphia: J.D. Lippincott.

Press, I. (2004) The measure of Quality, Quality Management in Health Care, 13 (4) 202-209.

Reed, V. and Woods, P. (2000) Manual of The Behavioural Status Index (BSI); a 'life skills' assessment for monitoring therapy in health care. United Kingdom: Psychometric Press.

Ross, T., Woods, P., Reed, V., Sookoo, S., Dean, A., Kettles, A., Almvik, R., Ter Horst, P., Brown, I., Collins, M., Walker, H. and Pfafflin, F. (2008) Assessing living skills in forensic mental health care with the behavioural status index: A European network study. Psychotherapy Research, 18 (3) 334-344.

Ross, T, Reed, V, Fontao, M, Pfafflin, F. (2012). Assessing reliability, validity, and clinical utility of the BEST-Index in measuring living skills among forensic inpatients. International Journal of Offender Therapy and Comparative Criminology, 56 (3) 385-400.

Scottish Government (2012) Mental Health Strategy (2012-2015). Edinburgh: Scottish Government.

Scottish Executive Health Department (2006) Rights Relationships and Responsibilities. Edinburgh: SEHD.

Scottish Executive Health Department (2010) Rights Relationships and Responsibilities Revised. Edinburgh: SEHD.

SPSS Inc. (2012) Statistical Package for Social Sciences version 19. SPSS Inc.

Sukkoo, S., Reed, V., Brown, I., Ross, T. (2007) Cognitive-attitudinal aspects of key-worker's talk about their patients in forensic psychiatric institutions. International Journal of Psychiatric Nursing Research, 12 (2) 1446-1458.

Świtaj, P., Anczewska, M., Chrostek, A., et al. (2012) Disability and schizophrenia: a systematic review of experienced psychosocial difficulties. BMC Psychiatry,12:193.

Taylor, C. and Gibbs, G.R. (2010) What is qualitative data analysis (QDA)? Online QDA website, [onlineqda.hud.ac.uk/Intro_QDA/what_is_qda.php]

Thomson, L.D.G., Bogue, J.P., Humphreys, M.S. and Johnstone, E.C. (1997) The State Hospital Survey: a description of psychiatric patients in conditions of special security in Scotland. Journal of Forensic Psychiatry, 8 (2) 263-284.

Turkington, D. and Bryant, C. (2007) 'How Cognitive Behavioural Therapy may improve social functioning in schizophrenia'. Progress in Neurology and Psychiatry, 11 (3) 19-21.

Vojt, G., Slesser, M., Marshall, L and Thomson, L. (2011) The clinical reality of implementing formal risk assessment and management measures within high secure forensic care. Medicine, Science and Law, 51, 220-227. DOI:10.1258/msl.2011.010149 
Walker, H., Tulloch, L., Ramm, M., Drysdale, E., Steele, A., MacPherson, G., Martin, C., Connaughton, J. (2013) A randomised controlled trial to explore insight into psychosis; the effects of a psychoeducation programme on insight in a Scottish forensic population. Journal of Forensic Psychiatry and Psychology, 24 (6) 756-771.

Webster, C.D., Douglas, K., Eaves, D. and Hart, S. (1997) HCR-20; Assessing the risk for violence (2 ${ }^{\text {nd }}$ ed.). Vancouver: Mental Health, Law, and Policy Institute, Simon Fraser University.

Webster, C.D., Martin, M.L., Brink, J., Nicholls, T.L. \& Desmarais, S. (2009) Manual for the Short-Term Assessment of Risk and Treatability (START) (Version 1.1). Port Coquitlam, BC: Forensic Psychiatric Services Commission and St. Joseph's Healthcare.

Woods, P., Reed, V. and Robinson, D. (1999) The Behavioural Status Index: therapeutic assessment of risk, insight, communication and social skills. Journal of Psychiatric and Mental Health Nursing, 6, 7990.

Woods, P., Reed, V. and Collins, M. (2001a) Measuring Communication and Social Skills in a High Security Forensic Setting using the Behavioural Status Index. The International Journal of Psychiatric Nursing Research, 7 (1) 760-777.

Woods, P., Reed. V. and Collins, M. (2001b) Measuring Insight in a High-security Forensic Setting Using the Behavioural Status Index. The British Journal of Forensic Practice, 3 (3) 3-12.

Woods, P. and Almvik, R. (2002) The Broset Violence Checklist (BVC). Acta Psychiatrica Scandinavica. 106,103-105.

Woods P., Reed, V., Collins, M. (2003) Exploring core relationships between insight and communication and social skills in mentally disordered offenders. Journal of Psychiatric and Mental Health Nursing, 10, 518-525. 


\section{Box 1: BEST-Index sub-scales}

- Communication and social skills - items illustrate adaptive social behaviours and processes by which interpersonal relationships are established

- Insight - examines the individual's cognitive constructs of reality, drawing on psychodynamic, cognitive-behavioural and phenomenological perspectives;

- Work and recreational activities - items cover a range of constructive activities, not necessarily paid work

- Self and Family Care - assess daily socially important areas such as personal hygiene, cooking, other aspects of self-care and care for other family or group members

- Empathy - assesses ability to conceptualise others' worlds through identified behaviours

- Risk - this scale is an addendum designed for use in forensic populations.

Table 1: Comparison of baseline and 18 month follow up results for BEST-Index subscales total scores

\begin{tabular}{|c|c|c|c|c|}
\hline Sub scale & $\begin{array}{l}\text { T1 baseline } \\
\text { mean, standard } \\
\text { deviation }\end{array}$ & $\begin{array}{l}\text { T2 post mean, } \\
\text { standard } \\
\text { deviation }\end{array}$ & Level of significance & Effect size \\
\hline Risk & $\begin{array}{l}n=160, m=85.1 \\
s d=14.11\end{array}$ & $\begin{array}{l}n=160,87.4 \\
s d=11.86\end{array}$ & $\begin{array}{l}T=-2.27, d f=159, \\
p=.024^{*}\end{array}$ & .18 \\
\hline Insight & $\begin{array}{l}n=159, m=60.4 \\
s d=19.6\end{array}$ & $\begin{array}{l}n=159, m=65.6 \\
s d=19.1\end{array}$ & $\begin{array}{l}T=-3.42, d f=158 \\
p=.001^{* *}\end{array}$ & .28 \\
\hline Communication & $\begin{array}{l}n=154, m=110.72 \\
s d=22.24\end{array}$ & $\begin{array}{l}n=154, m=114.18 \\
s d=23.22\end{array}$ & $\begin{array}{l}T=-1.87, d f=153 \\
p=.062 \mathrm{NS}\end{array}$ & \\
\hline $\begin{array}{l}\text { Work and } \\
\text { Recreation }\end{array}$ & $\begin{array}{l}n=153, m=57.24 \\
s d=25.04\end{array}$ & $\begin{array}{l}n=153, m=64.45 \\
s d=25.69\end{array}$ & $\begin{array}{l}T=-3.14, d f=152, \\
p=.002^{* *}\end{array}$ & .29 \\
\hline Self care & $\begin{array}{l}n=151, m=87.91 \\
s d=38.94\end{array}$ & $\begin{array}{l}n=151, m=94.51, \\
s d=36.96\end{array}$ & $\begin{array}{l}T=-1.78, d f=150, \\
p=.077 \mathrm{NS}\end{array}$ & \\
\hline Empathy & $\begin{array}{l}n=149, m=96.18 \\
s d=44.89\end{array}$ & $\begin{array}{l}n=149, m=93.79 \\
s d=26.48\end{array}$ & $\begin{array}{l}T=.65, d f=148, p=.514 \\
\text { NS }\end{array}$ & \\
\hline
\end{tabular}

${ }^{* *}$ statistically significant at .01 level ; ${ }^{*}$ statistically significant at 0.05 level 
Table 2: Comparison between TSH and MRH BEST-Index scores at baseline and 18 month follow up

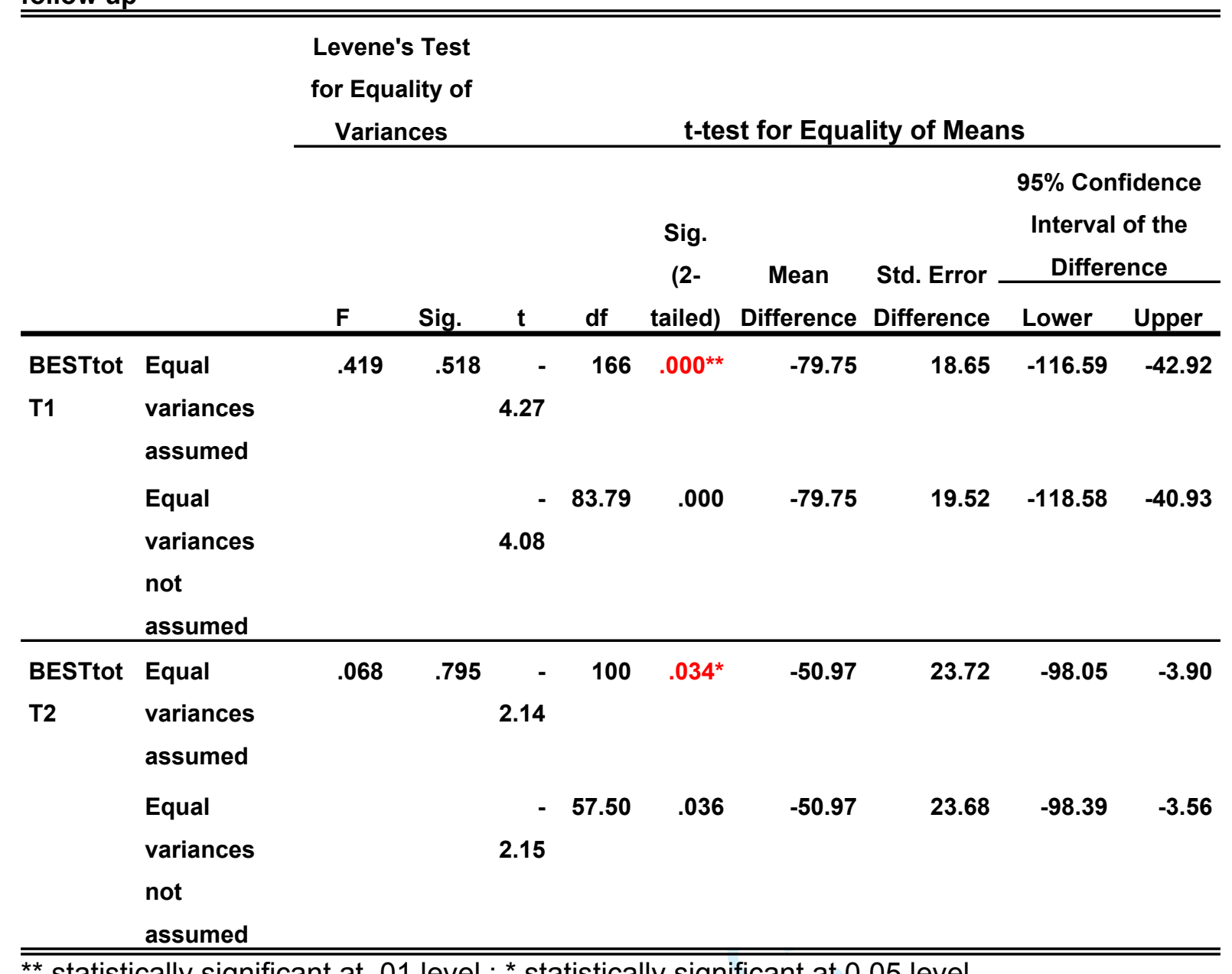

Figure 1

Best -Index scoring. Item 8, risk subscale

\begin{tabular}{|l|l|l|l|l|l|}
\hline \multicolumn{6}{|l|}{ Item 8: Verbal Aggression without apparent trigger event } \\
\hline Description & $\begin{array}{l}\text { Usually } \\
\text { verbally } \\
\text { aggressive } \\
\text { without } \\
\text { apparent } \\
\text { trigger event }\end{array}$ & $\begin{array}{l}\text { Frequently } \\
\text { verbally } \\
\text { aggressive } \\
\text { without } \\
\text { apparent trigger } \\
\text { event }\end{array}$ & $\begin{array}{l}\text { Occasionally } \\
\text { verbally } \\
\text { aggressive } \\
\text { without } \\
\text { apparent trigger } \\
\text { event }\end{array}$ & $\begin{array}{l}\text { Rarely verbally } \\
\text { aggressive } \\
\text { without } \\
\text { apparent trigger } \\
\text { event }\end{array}$ & $\begin{array}{l}\text { No evidence of } \\
\text { verbal } \\
\text { aggression } \\
\text { whilst in } \\
\text { hospital }\end{array}$ \\
\hline Score & $\mathbf{1}$ & $\mathbf{2}$ & $\mathbf{3}$ & $\mathbf{4}$ & $\mathbf{5}$ \\
\hline
\end{tabular}


Figure 2

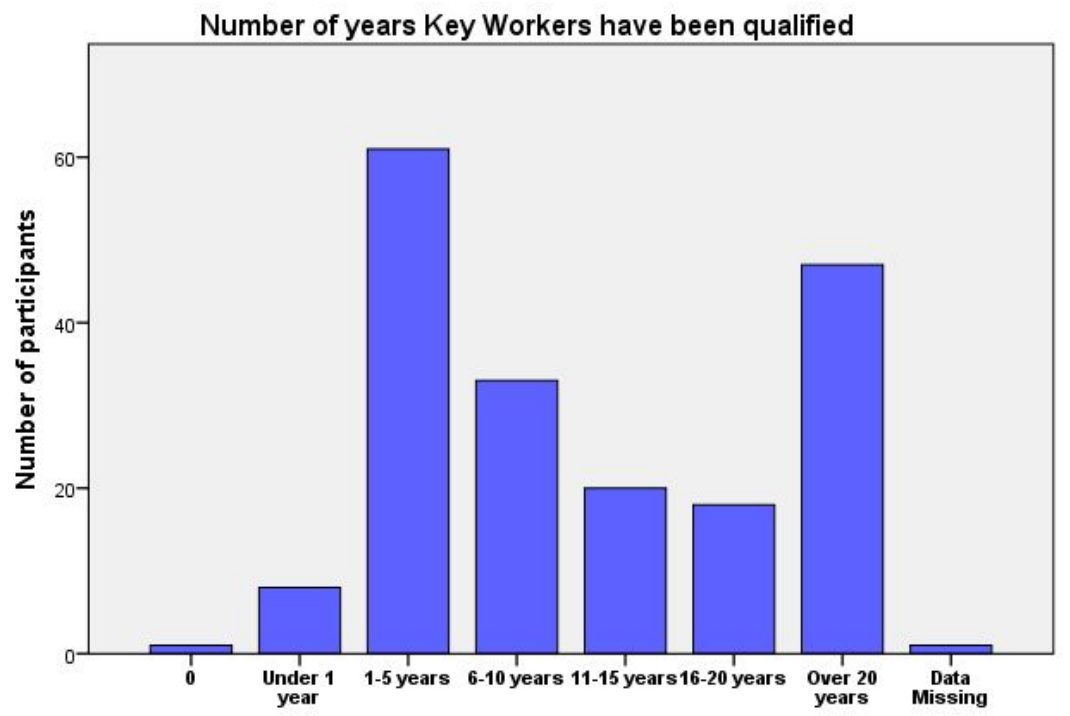


Figure 3

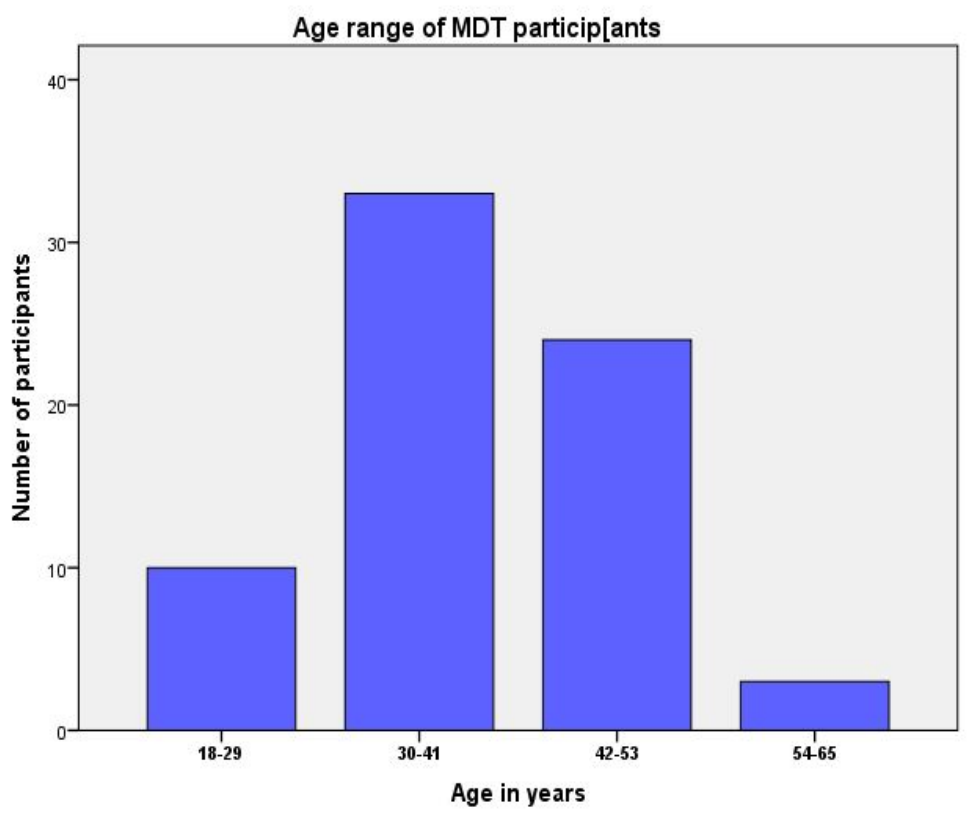

Figure 4

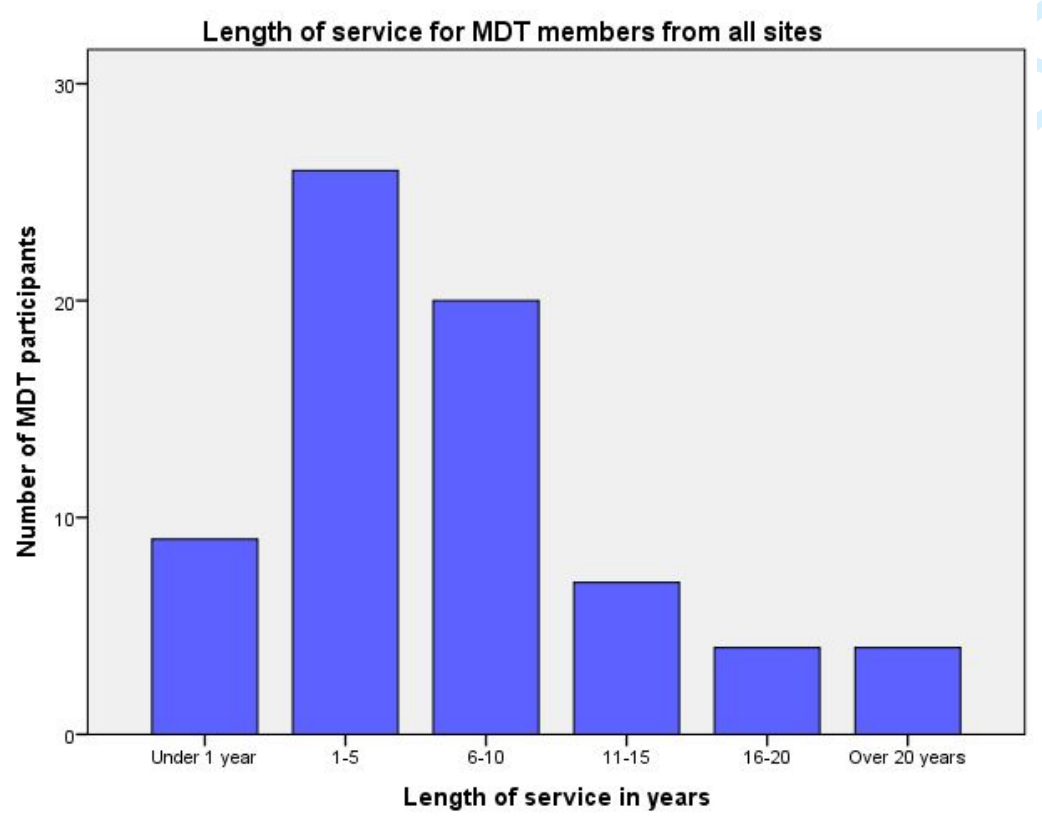

